\title{
A Generalized Tunneling Formula for Quantum Device Modeling
}

\author{
R. LAKE ${ }^{a}$, G. KLIMECK ${ }^{a, b}$, R. C. BOWEN ${ }^{b}$, D. JOVANOVIC ${ }^{a}$, P. SOTIRELIS ${ }^{a}$ and W. R. FRENSLEY \\ ${ }^{\mathrm{a}}$ Corporate R\&D, Texas Instruments Incorporated, Dallas, TX 75265, ${ }^{\mathrm{b}}$ Erik Jonsson School of Engineering and Computer Science, The Uni- \\ versity of Texas at Dallas, Richardson, TX 75083
}

\begin{abstract}
The generalized tunneling formula with the simple model for the broadening in the contacts gives surprisingly good results for the majority of RTD structures. It is just as fast as the standard coherent tunneling simulators and much more versatile. It is easily generalized to multi-band and multidimensional models. The multi-band generalization of the theory and the effect of the optical potential are described.
\end{abstract}

Keywords: resonant tunneling; quantum device modeling

The theory of the generalized open system boundary conditions $[1,2]$ leads to a generalized tunneling formula which is the basis for our quantum device simulator $[3,4]$. The generalized boundary conditions allow one to take a large structure and partition it into two large reservoirs and a short device provided that the reservoir regions are well equilibrated with the $n^{+}$ contacts (see Fig. (1a)). These defined reservoirs have a complicated density of states which must be calculated numerically. The density of states or spectral function is displayed by the gray-scale in Fig. (1a). The partitioning of the system serves two purposes: (1) it allows one to inject into the device from quantized emitter states in a coherent tunneling formula [5], and (2) it reduces the device domain for the computationally intensive calculations required to include scattering.

The extension of the previous discussions $[1,2]$ based on the single band tight binding model is straightforward. The scalar quantities become block matrices in a localized orbital multi-band model.
Using the standard $s p^{3} s^{*}$ basis [6] the current equation for a device defined between and $\Re$ including layers 1 through $\mathrm{N}$ becomes

$$
J_{L}=\frac{2 e}{\hbar A} \sum_{k} \int \frac{d E}{2 \pi} \operatorname{tr}\left\{\Gamma_{1,1}^{B L} G_{1, N}^{R} \Gamma_{N, N}^{B R} G_{N, 1}^{A}\right\}\left(f_{L}-f_{R}\right)
$$

where the $\Gamma$ 's include the effect of the states in the reservoirs, $f_{\mathcal{L}(\Re)}$ is the Fermi factor of the left (right) contact. The $\Gamma$ 's and $G$ 's are block matrices of dimension equal to the number of orbitals per layer and the trace is over the orbital indices. The subscripts refer to the layer number.

To calculate the Green function, we use the example of a three layer device, $\{i, j\} \in\{1, \ldots, 3\} ; G^{R}$ is found from

$$
\left[G^{R}\right]=\left[\begin{array}{ccc}
E-D_{1}-\sum_{1,1}^{R B} & t_{1,2} & 0 \\
t_{1,2} & E-D_{2} & t_{2,3} \\
0 & t_{3,2} & E-D_{3}-\sum_{3,3}^{R B}
\end{array}\right]^{-1}
$$



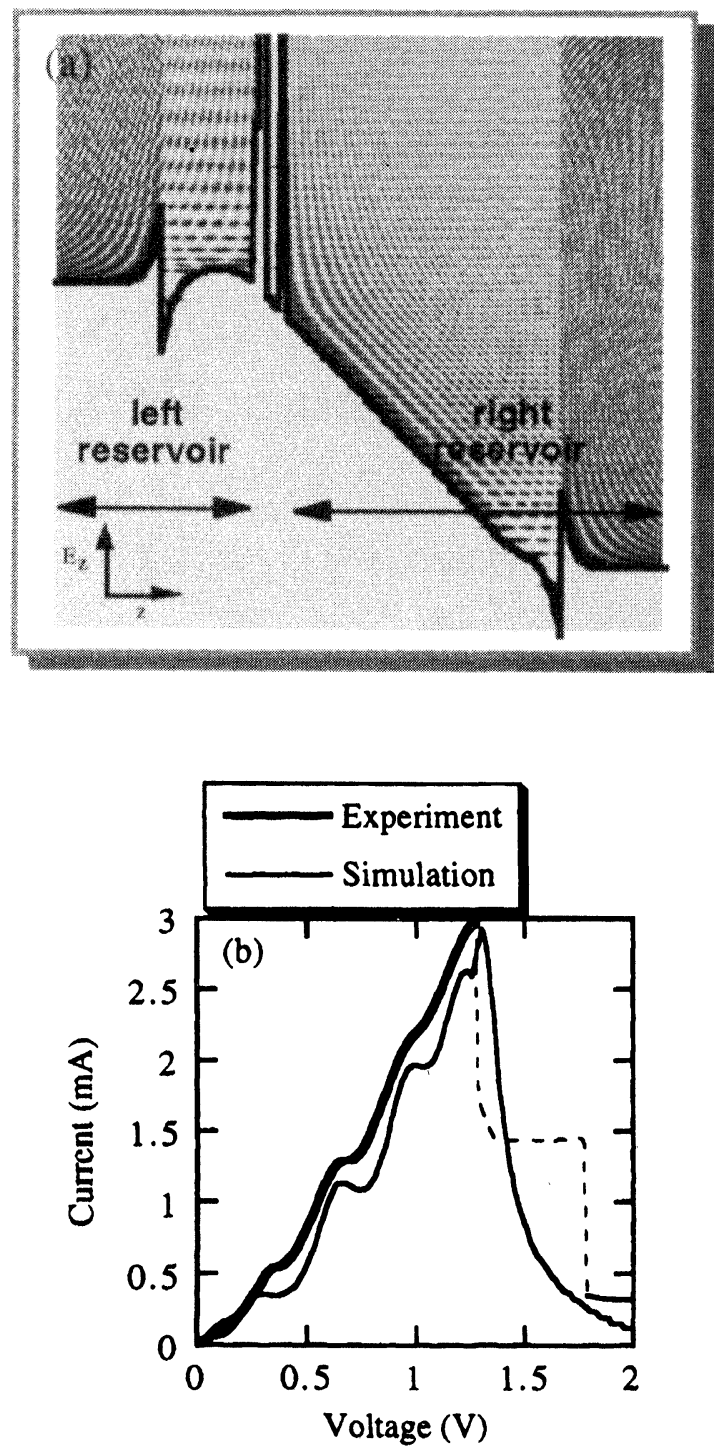

FIGURE 1 (a) Conduction band profile and corresponding density of states for a device with many quantized emitter states. (b) Corresponding experimental and simulated I-V characteristic

where $\Sigma_{1,1}^{R B}=t_{1,0} g_{0,0}^{R} t_{0,1} \quad$ and $\Gamma_{1,1}^{B}=t_{1,0} i\left[g_{0,0}^{R}\right.$ $\left.-g_{0,0}^{A}\right] t_{0,1}$. All of the symbols above represent block matrices of dimension equal to the number of orbitals per layer. $g_{0,0}^{R}$ is the surface Green function for the semi-infinite region ending at layer 0 immediately to the left of the device.
Starting with the bulk surface Green function in the flat band region of the left lead [7], the surface Green function at layer 0 is calculated with the recursive Green function method. Using our boundary self energy, $\Sigma_{i, i}^{R B}$, we have the recursive Green function algorithm

$$
g_{L, L}^{R}=\left[E-D_{L}-t_{L, L-1} g_{L-1, L-1}^{R} t_{L-1, L}\right]^{-1}
$$

An imaginary potential, $i \eta$, is included in the diagonal Hamiltonian matrix elements of the contact regions to account for the effects of scattering induced broadening of any quantized states that form there.

The power of this approach is demonstrated in Fig. (1). Such a device can now be treated within the framework of a single electron tunneling formula, Eq. (1). We have obtained similar results in a multi-band $s p^{3} s^{*}$ calculation.

The key that makes the approach work is the optical potential included in the contacts. What is its effect ? For devices with 3D emitters, ie. where there is no quantization in the emitter, the effect is small. For such a device, the conduction band profile, spectral function, and I-V for 4 different values of $\eta$ is shown in Fig. (2). As $\eta$ increases, the peak current is slightly decreased and the valley current is slightly increased.

For structures with both $2 \mathrm{D}$ states in the emitter and 2D states in the device, the effect is large. The I-V calculated with 4 different values of $\eta$ corresponding to the device of Fig. (1) is shown in Fig. (3). As $\eta$ is reduced, the I-V becomes a series of narrow peaks whose width is finally determined be the intrinsic linewidth of the resonances. The necessity of including broadening factors has been observed before, 5 meV for Turley et al. [8] and 0.2 -16 meV for Chen et al. [9]. For such devices, the optical potential in the contacts covers over a number of omissions : (1) Correct numerical integration over the transverse momentum [10]. Initial calculations indicate that the value of $\eta$ can be reduced by a factor of $1 / 2$ when the transverse integration is done correctly. (2) The broadening of the device states due to scattering, and (3) the actual broadening of the emitter states due to scattering. Note well that as the optical potential model is improved, everything else must be improved 

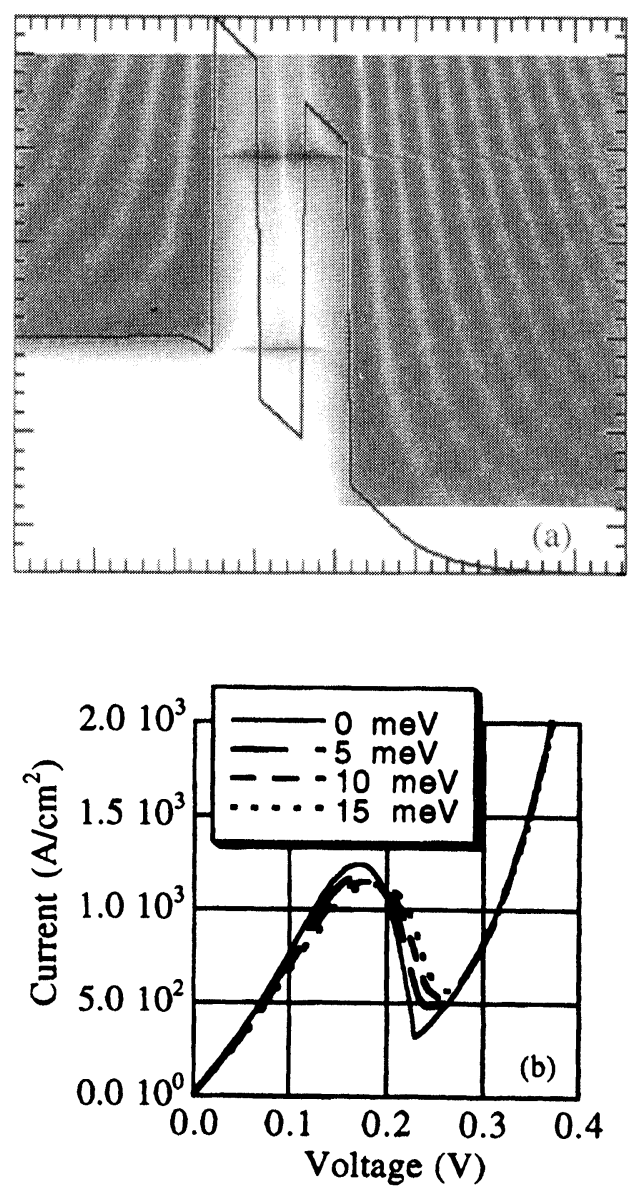

FIGURE 2 (a) Conduction band profile and corresponding density of states for a device with a bulk 3D emitter. (b) I-V characteristic calculated with 4 different values of the optical potential

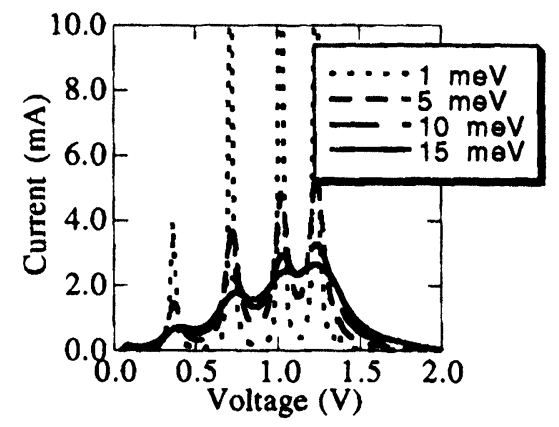

FIGURE $3 \mathrm{I}-\mathrm{V}$ characteristic calculated with 4 different values of the optical potential for the device of Fig. (1) to maintain the same accuracy of the results since the omissions that were covered by the simple model are no longer covered. Incremental improvements are difficult.

The generalized tunneling formula with the simple model for the broadening in the contacts gives surprisingly good results for the majority of RTD structures. It is just as fast as the standard coherent tunneling simulators [11] and much more versatile. It is also easily generalized to $2 \mathrm{D}$ and $3 \mathrm{D}$ simulators. It is useful as a fast design tool and as the starting point for more sophisticated calculations.

\section{References}

[1] R. Lake, in Procedings of the Third International Workshop on Computational Electronics (Portland, OR, May 18-20, 1994), pp. 239-242.

[2] R. Lake, in Quantum Transport in Ultrasmall Devices, NATO ASI Series, Vol. 342, edited by D. K. Ferry, H. L. Grubin, C. Jacoboni and A. Jaucho (Plenum Press, New York, 1995), pp. 521-524.

[3] G. Klimeck, R. Lake, R. C. Bowen, W. R. Frensley, and D. Blanks, in the 1995 53rd Annual Device Research Conference Digest, p. 52, Charlottesville, VA, June 19-21, 1995.

[4] G. Klimeck, R. Lake, R. C. Bowen, W. R. Frensley, and T. Moise, Appl. Phys. Lett. 672639 (1995).

[5] T. Fiig and A. Jauho, Surf. Sci. 267, 392 (1992).

[6] J. A. Stovneng and P. Lipavsky, Phys. Rev. B 49, 16494 (1991).

[7] R. C. Bowen, W. R. Frensley, G. Klimeck, and R. K. Lake, Phys. Rev. B 52, 2754 (1995).

[8] P. J. Turley et al., Phys. Rev. B 47, 12640 (1993).

[9] J. Chen, C. H. Yang, and R. A. Wilson, J. Appl. Phys. 71, 1537 (1992).

[10] T. B. Boykin, R. E. Carnahan, and K. P. Martin, Phys. Rev. B 51, 2273 (1995).

[11] D. Landheer and G. C. Aers, Superlattices and Microstructures 7, 17 (1990).

\section{Biographies}

Roger Lake is a Member Technical Staff at Raytheon TI Systerns. He is working on scattering, disorder, and optical processes in III-V and Silicon quantum well semiconductor devices.

Gerhard Klimeck performed this work as a postdoc at the University of Texas at Dallas and later as a Member Technical Staff at Texas Instruments Incorporated. He will move to the Jet Propulsion Laboratory in early 1998. His research interest is the modeling of electron transport in nanoelectronic devices. 
R. Chris Bowen performed this work as a graduate student at the University of Texas at Dallas and he is presently Member Technical Staff at Texas Instruments Incorporated. His current research interest is full-band modeling of quantum electron transport in Silicon and III-V structures.

Dejan Jovanovic performed this work as a Member Technical Staff at Texas Instruments Incorporated and he is presently a member technical staff at Motorola. His research interests include quantum mechanical modeling of semiconductor devices.
Paul Sotirelis performed this work as a technical consultant under contract with Texas Instruments Incorporated and he is presently the Computational Electronics and Nanoelectronics On-Site Lead at the ASC-MSRC, one of the four large DoD high performance computing centers. His research interests include modeling novel quantum semiconductor devices.

William R. Frensley is Professor and Head of the Electrical Engineering Program at the University of Texas at Dallas. His research interests include theory and simulation of high-performance semiconductor devices. 

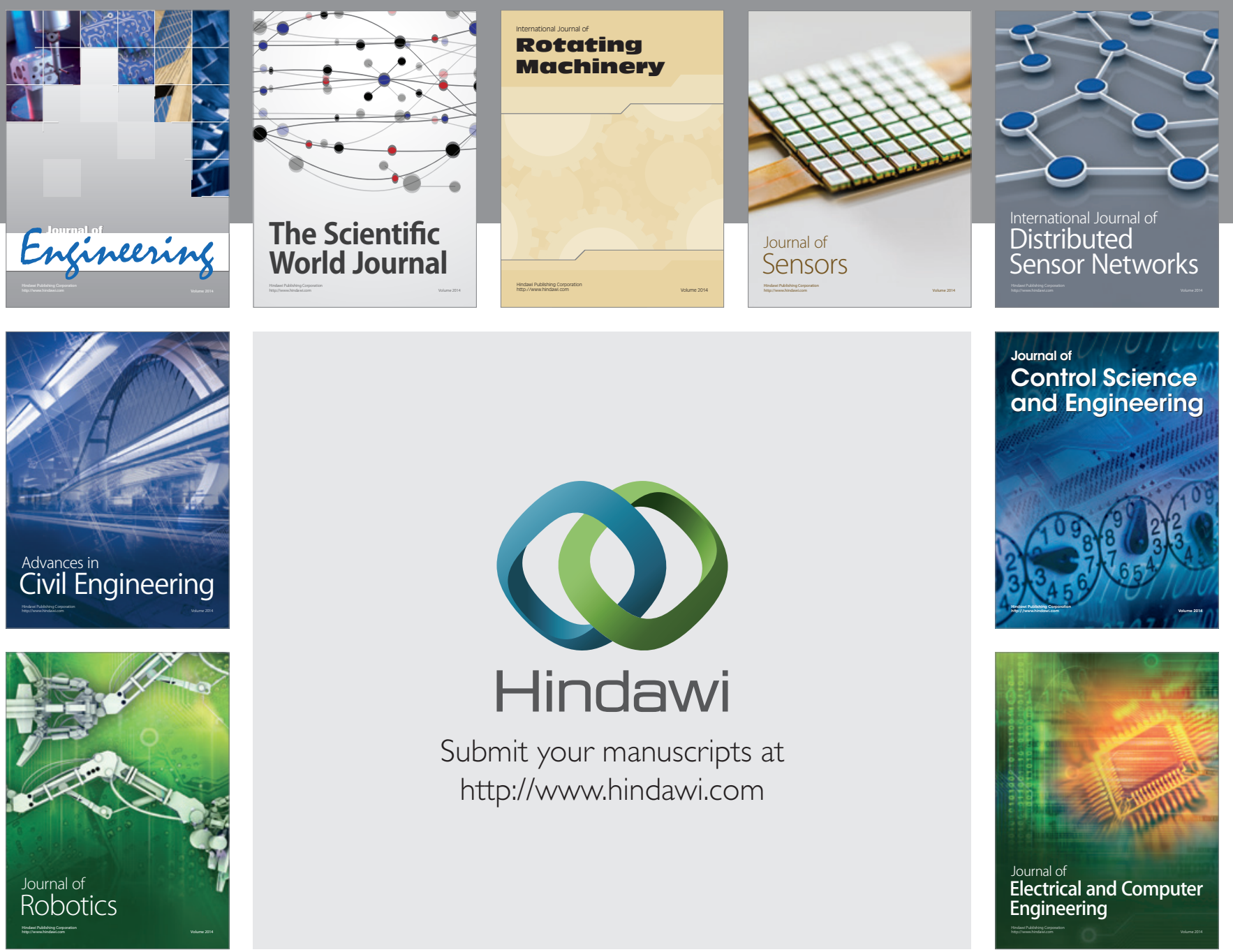

Submit your manuscripts at

http://www.hindawi.com
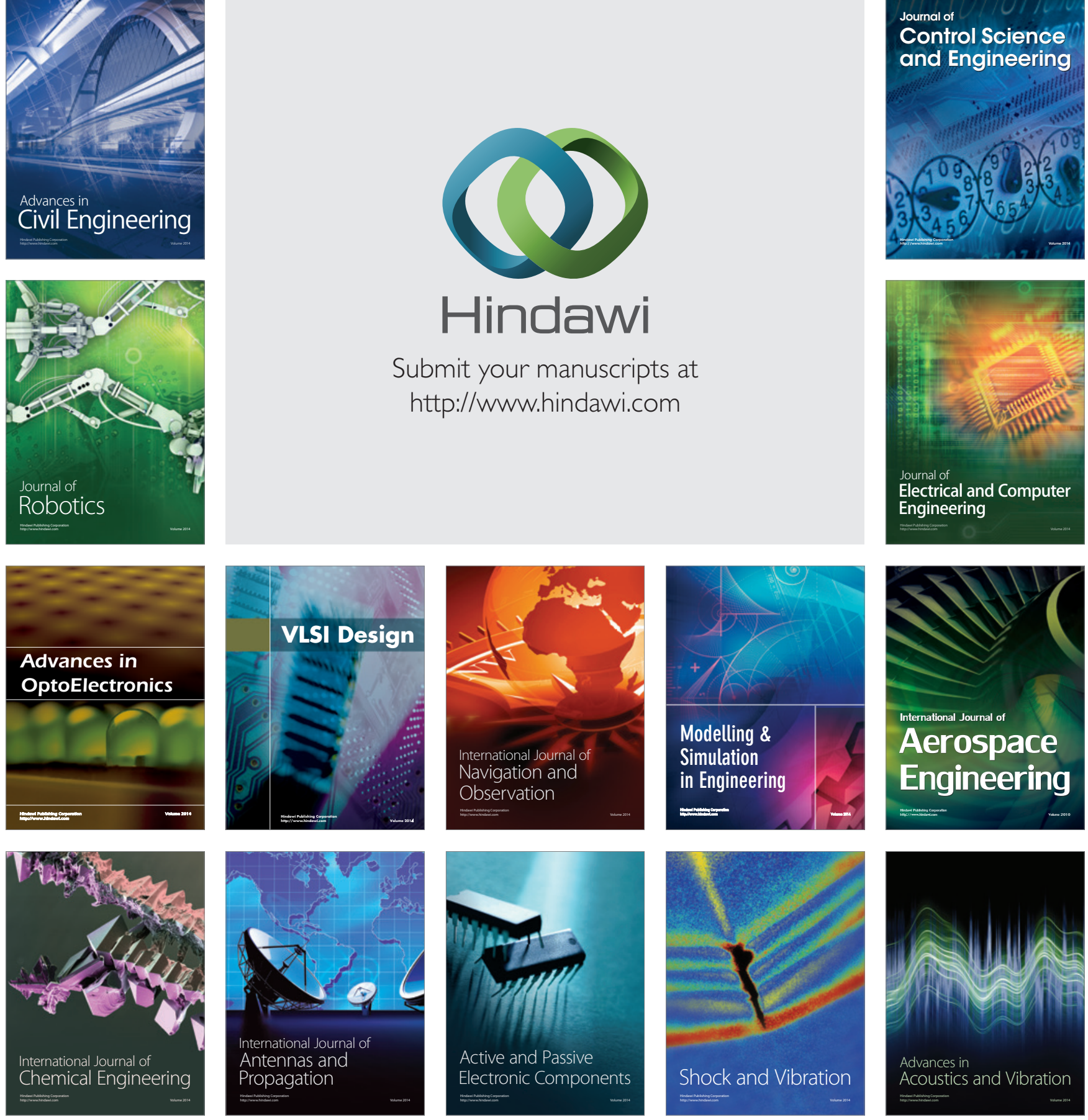